\title{
A SIMPLIFIED APPROACH TO THE NUMERICAL DESCRIPTION OF METHANE-AIR DIFFUSION FLAMES
}

\author{
MARCUS BOLLIG, ${ }^{1}$ AMABLE LIÑÁN, ${ }^{1}$ ANTONIO L. SÁNCHEZ, ${ }^{2}$ AND FORMAN A. WILLIAMS ${ }^{3}$ \\ ${ }^{1}$ Departamento de Motopropulsión y Termofluidodinámica \\ E. T. S. I. Aeronáuticos \\ Universidad Politécnica de Madrid \\ 28040 Madrid, Spain \\ ${ }^{2}$ Area de Mecánica de Fluidos \\ Escuela Politécnica Superior \\ Universidad Carlos III de Madrid \\ 28911 Leganés, Spain \\ ${ }^{3}$ Center for Energy and Combustion Research \\ University of California San Diego \\ La Jolla, CA 92093-0411, USA
}

\begin{abstract}
Starting with a three-step reduced chemistry description that employes $\mathrm{H}_{2}$ and $\mathrm{CO}$ as the only intermediates not in steady state, a simplified formulation aimed at facilitating numerical computations of nonpremixed methane-air systems is developed. The analysis retains finite rates for radical recombination and $\mathrm{CO}$ oxidation but assumes infinitely fast fuel consumption taking place in a diffusion-controlled manner in an infinitely thin reaction sheet. To remove stiffness associated with the fast fuel consumption, the conservation equations for the major species and the temperature are written in terms of generalized coupling functions that for predictive accuracy permit species diffusivities that differ from the thermal diffusivity. The resulting formulation, which automatically determines the position of the fuel-consumption layer without necessity of front tracking or further interface approximations, can be used for analytical, computational, and modeling studies of both laminar and turbulent flows, removing stiffness difficulties associated with highly disparate chemical time scales. Comparisons of results of the simplified formulation in the counterflow mixing layer with those obtained with detailed chemistry and transport descriptions indicate that the proposed formulation applies with good accuracy to strain conditions ranging from weakly strained, robust flames to near-extinction flames.
\end{abstract}

\section{Introduction}

Analyses of chemically reacting flows with the full chemical kinetics of combustion are prohibitively complicated except in the simplest geometrical configurations. For this reason, in recent years, a great effort has been placed on obtaining descriptions that are realistically based on known elementary chemical reaction rates yet sufficiently simple to be used for complicated flows. The general approach has been to begin with the conservation equations in a selected form and to graft the chemical-kinetic approximations on top of them, resulting in formulations that are increasingly difficult to apply as the chemistry is allowed to become more complex. The present work adopts the opposite procedure; that is, the starting point is the reduced chemical description, and the correspondingly appropriate forms of the conservation equations are then derived. In this manner, formulations can be obtained that are well suited for describing a wide range of combustion problems efficiently and with sufficient fidelity.
The first step in this direction for diffusion flames was taken by Burke and Schumann [1], who considered the reaction-sheet approximation for the chemistry and introduced a continuously differentiable field variable that measures the fuel concentration on the fuel side of the sheet and the oxidizer concentration on the oxidizer side. In their formulation, they considered diffusivities for reactants and for heat to be equal (unity Lewis numbers), a simplifying approximation that enables the fuel and oxidizer concentrations as well as the temperature to be expressed in terms of a single coupling function, which becomes the mixture-fraction variable when normalized to have the values zero and unity in the oxidizer and fuel streams, respectively.

This formulation has been recently generalized to allow for constant values of the Lewis numbers of the reactants that differ from unity, with the proviso that the specific heat at constant pressure for the gas mixture is constant [2]. Two mixture-fraction variables are helpful for this generalization, the ordinary one and one in which the species concentrations are 
weighted by their diffusion coefficients. In addition, an excess-enthalpy variable is needed to describe the temperature field when the Lewis number of the reactants is different from unity, because enthalpies then are not related uniquely to a mixture fraction, and a differential equation describing energy conservation must be retained [2]. It is nevertheless possible to define continuous and sufficiently differentiable generalized coupling functions that serve as field variables in the conservation equations of the formulation, enabling analyses to proceed without paying special attention to the location or structure of the front representing the reaction sheet.

These formulations apply for the limit in which the rates of all elementary chemical steps are fast in comparison with flow rates and diffusion rates. Real chemistry involves a number of steps, some fast and others much slower, thereby introducing stiffness into the conservation equations and causing difficulties in numerical solutions. The present objective is to circumvent such difficulties by generalizing the Burke-Schumann formulation to allow for finiterate slow chemistry in methane-air flames, an objective that was recently achieved for hydrogen-oxygen diffusion flames [3]. It was found that, in such flames, fast two-body radical chain-branching steps lead to the overall radical-production reaction $3 \mathrm{H}_{2}$ $+\mathrm{O}_{2} \rightarrow 2 \mathrm{H}_{2} \mathrm{O}+2 \mathrm{H}$ being infinitely fast and therefore occurring at a modified Burke-Schumann reaction sheet, while the overall recombination $2 \mathrm{H} \rightarrow$ $\mathrm{H}_{2}$, occurring at the rates of the elementary threebody steps $\mathrm{H}+\mathrm{H}+\mathrm{M} \rightarrow \mathrm{H}_{2}+\mathrm{M}$ and $\mathrm{H}+\mathrm{O}_{2}$ $+\mathrm{M} \rightarrow \mathrm{HO}_{2}+\mathrm{M}$, is much slower and in the formulation can proceed in a distributed manner throughout the flow field. The resulting generalized Burke-Schumann formulation remains quite accurate over about 3 orders of magnitude in strain time for this system and describes the smooth transition from high-strain, near-extinction conditions, in which three hydrogen molecules are consumed for each oxygen molecule at the reaction sheet, according to the rapid overall radical-production step, to low-strain, near-equilibrium conditions, in which instead only two hydrogen molecules are consumed, according to $2 \mathrm{H}_{2}+\mathrm{O}_{2} \rightarrow 2 \mathrm{H}_{2} \mathrm{O}$, the overall chemistry in the classical Burke-Schumann treatment.

The detailed combustion chemistry of hydrocarbon fuels is much more complicated than that of hydrogen. Because hydrocarbon-air diffusion flames nevertheless are of importance in numerous applications, for these fuels there is strong motivation to try to develop the type of generalized Burke-Schumann formulation that was found [3] for hydrogenoxygen non-premixed systems. Methane is the simplest hydrocarbon fuel, and therefore, the present first step in this direction addresses methane-air diffusion flames. Although methane is of interest in itself, as the main component of natural gas, it may be even more important as a model alkane. Especially at the three-step reduced chemical-kinetic description employed here, formulations for all alkanes can readily parallel that for methane [4]. The generalized Burke-Schumann formulation developed here, therefore, will be simply extendible to other alkanes and, indeed, quite likely to hydrocarbon-air diffusion flames in general.

\section{Reduced Chemical-Kinetic Mechanism for Methane-Air Diffusion Flames}

It has been well established that a good four-step reduced-chemistry description for methane-air flames can be written in overall form as [5]

$$
\begin{gathered}
\mathrm{CH}_{4}+2 \mathrm{H}+\mathrm{H}_{2} \mathrm{O} \rightleftharpoons \mathrm{CO}+4 \mathrm{H}_{2} \\
\mathrm{CO}+\mathrm{H}_{2} \mathrm{O} \rightleftharpoons \mathrm{CO}_{2}+\mathrm{H}_{2} \\
3 \mathrm{H}_{2}+\mathrm{O}_{2} \rightleftharpoons 2 \mathrm{H}_{2} \mathrm{O}+2 \mathrm{H} \\
2 \mathrm{H} \rightleftharpoons \mathrm{H}_{2}
\end{gathered}
$$

This description involves the introduction of steadystate approximations for all radicals except the $\mathrm{H}$ atom, so that radical concentrations in the flame are related to that of H. As shown in Ref. [6], flame structures and extinction strain rates of counterflow diffusion flames, calculated numerically on the basis of this four-step description, agree well with those calculated with full detailed chemistry and also with those found experimentally.

There are some aspects of methane-air diffusion flames for which the foregoing reduced chemistry is inaccurate. For example, rates of production of oxides of nitrogen depend in part on the prompt mechanism, which can be included with reasonable accuracy only if a five-step mechanism is employed [7], with account taken of the formation and consumption of $\mathrm{C}_{2} \mathrm{H}_{2}$, an additional species not in steady state. Most aspects of methane-air diffusion can, however, be described well with the reduced mechanism I'-IV'. In particular, contrary to predictions of simple one-step Arrhenius approximations, $\mathrm{O}_{2}$ leaks through the flame, but $\mathrm{CH}_{4}$ does not, and this result is achieved by the separate fuel-consumption $\left(\mathrm{I}^{\prime}\right)$ and oxygen-consumption (III') steps because the removal of radicals by $\mathrm{I}^{\prime}$ prevents the oxygen from being consumed on the fuel side. A three-step overall approximation with infinitely fast fuel consumption is employed in the present work, which relinquishes this last attribute of the four-step description.

Introducing a steady-state approximation for $\mathrm{H}$, an approximation that is seen to be increasingly accurate as the pressure increases from atmospheric conditions [8-10], reduces $\mathrm{I}^{\prime}-\mathrm{IV}^{\prime}$ to three overall reactions:

$$
\mathrm{CH}_{4}+\mathrm{O}_{2} \rightarrow \mathrm{CO}+\mathrm{H}_{2}+\mathrm{H}_{2} \mathrm{O}
$$




$$
\begin{gathered}
\mathrm{CO}+\mathrm{H}_{2} \mathrm{O} \rightleftharpoons \mathrm{CO}_{2}+\mathrm{H}_{2} \\
2 \mathrm{H}_{2}+\mathrm{O}_{2} \rightarrow 2 \mathrm{H}_{2} \mathrm{O}
\end{gathered}
$$

which represent the starting scheme in the present investigation. To obtain a generalized Burke-Schumann description, conditions are addressed under which step I can be approximated as being infinitely fast, although initially it will be ascribed the irreversible rate derived from systematically simplified reduced chemistry [8]. The principal rates (moles per unit volume per unit time) $\omega_{\mathrm{I}}, \omega_{\mathrm{II}}$, and $\omega_{\mathrm{III}}$ of the three overall reactions then become

$$
\begin{gathered}
\omega_{\mathrm{I}}=k_{1 f}\left[\mathrm{CH}_{4}\right][\mathrm{H}] \\
\omega_{\mathrm{II}}=k_{2 f}[\mathrm{CO}][\mathrm{OH}]-k_{2 b}\left[\mathrm{CO}_{2}\right][\mathrm{H}] \\
\omega_{\mathrm{III}}=k_{3 f}\left[\mathrm{O}_{2}\right][\mathrm{H}][\mathrm{M}]
\end{gathered}
$$

where $[i]$ denotes the concentration of chemical species $i, M$ representing a third body, and $k_{j f}$ and $k_{j b}$ the specific reaction-rate constants in the forward and backward directions for the elementary reactions $\mathrm{CH}_{4}+\mathrm{H} \stackrel{1}{\rightarrow} \mathrm{CH}_{3}+\mathrm{H}_{2}, \mathrm{CO}+\mathrm{OH} \stackrel{2}{\rightleftharpoons} \mathrm{CO}_{2}$ $+\mathrm{H}$, and $\mathrm{O}_{2}+\mathrm{H}+\mathrm{M} \stackrel{3}{\rightarrow} \mathrm{HO}_{2}+\mathrm{M}$. Note that third-body efficiencies must be taken into account for increased accuracy when computing [M]. Appropriate simplified steady-state expressions for the concentrations of $\mathrm{H}$ and $\mathrm{OH}$, necessary in evaluating equation 1 , are given by

$$
[\mathrm{H}]=\left(1-\frac{k_{3 f}[M]}{k_{4 f}}\right)^{1 / 2} K_{\mathrm{H}}\left[\mathrm{O}_{2}\right]^{1 / 2}\left[\mathrm{H}_{2}\right]^{3 / 2} /\left[\mathrm{H}_{2} \mathrm{O}\right]
$$

and

$$
[\mathrm{OH}]=\left(1-\frac{k_{3 f}[M]}{k_{4 f}}\right)^{1 / 2} K_{\mathrm{OH}}\left[\mathrm{O}_{2}\right]^{1 / 2}\left[\mathrm{H}_{2}\right]^{1 / 2}
$$

where $K_{\mathrm{H}}=1.27 \exp (2997 / T)$ and $K_{\mathrm{OH}}=5.97$ $\exp (-4696 / T)$ are equilibrium constants related to those of the shuffle reactions $\mathrm{O}_{2}+\mathrm{H} \stackrel{4}{\rightleftharpoons} \mathrm{OH}+\mathrm{O}$, $\mathrm{H}_{2}+\mathrm{O} \stackrel{5}{\rightleftharpoons} \mathrm{OH}+\mathrm{H}$, and $\mathrm{H}_{2}+\mathrm{OH} \stackrel{6}{\rightleftharpoons} \mathrm{H}_{2} \mathrm{O}+\mathrm{H}$ through $K_{\mathrm{H}}=K_{4}^{1 / 2} K_{5}^{1 / 2} K_{6}$ and $K_{\mathrm{OH}}=K_{4}^{1 / 2} K_{5}^{1 / 2}$. In deriving equations 2 and 3 , additional assumptions of partial equilibrium for the shuffle reactions 5 and 6 are made. It is also worth mentioning that a cutoff must be incorporated in computations when evaluating equations 2 and 3 , so that $[\mathrm{H}]=[\mathrm{OH}]=0$ for $T<T_{c}$, where $T_{c}$ is the crossover temperature of $\mathrm{H}_{2}-\mathrm{O}_{2}$ systems (at which $k_{3 f}[M]=k_{4 f}$ ).

\section{Conservation Equations}

It is convenient to work with the variables $\Gamma_{i}=$ $Y_{i} / W_{i}=[i] / \rho$, where $Y_{i}$ and $W_{i}$ denote the mass fraction and molecular weight for chemical species $i$, respectively, and $\rho$ is the density. If we let $D_{T}=\lambda$ / $\left(\rho c_{p}\right)$ denote the thermal diffusivity of the gas mixture, with $\lambda$ and $c_{p}$ representing its thermal conductivity and specific heat at constant pressure; then in a Fickian diffusion approximation for each species, their conservation equations can be written for the reduced chemistry I-III as

$$
\begin{aligned}
& \rho \frac{D}{D t}\left(\Gamma_{\mathrm{CH}_{4}}\right)-\nabla \cdot\left[\frac{\rho D_{T}}{L_{\mathrm{CH}_{4}}} \nabla \Gamma_{\mathrm{CH}_{4}}\right]=-\omega_{\mathrm{I}} \\
& \rho \frac{D}{D t}\left(\Gamma_{\mathrm{CO}_{2}}\right)-\nabla \cdot\left[\frac{\rho D_{T}}{L_{\mathrm{CO}_{2}}} \nabla \Gamma_{\mathrm{CO}_{2}}\right]=\omega_{\mathrm{II}} \\
& \rho \frac{D}{D t}\left(\Gamma_{\mathrm{CH}_{4}}-\Gamma_{\mathrm{O}_{2}}\right)
\end{aligned}
$$$$
-\nabla \cdot\left[\rho D_{T}\left(\frac{1}{L_{\mathrm{CH}_{4}}} \nabla \Gamma_{\mathrm{CH}_{4}}-\frac{1}{L_{\mathrm{O}_{2}}} \nabla \Gamma_{\mathrm{O}_{2}}\right)\right]=\omega_{\mathrm{III}}
$$

$$
\begin{aligned}
& \rho \frac{D}{D t}\left(\Gamma_{\mathrm{CH}_{4}}+\Gamma_{\mathrm{CO}}+\Gamma_{\mathrm{CO}_{2}}\right) \\
& -\nabla \cdot\left[\rho D _ { T } \left(\frac{1}{L_{\mathrm{CH}_{4}}} \nabla \Gamma_{\mathrm{CH}_{4}}+\frac{1}{L_{\mathrm{CO}}} \nabla \Gamma_{\mathrm{CO}}\right.\right. \\
& \left.\left.\quad+\frac{1}{L_{\mathrm{CO}_{2}}} \nabla \Gamma_{\mathrm{CO}_{2}}\right)\right]=0 \\
& \rho \frac{D}{D t}\left(\Gamma_{\mathrm{CH}_{4}}+\frac{1}{2} \Gamma_{\mathrm{H}_{2} \mathrm{O}}+\frac{1}{2} \Gamma_{\mathrm{H}_{2}}\right) \\
& -\nabla \cdot\left[\rho D _ { T } \left(\frac{1}{L_{\mathrm{CH}_{4}}} \nabla \Gamma_{\mathrm{CH}_{4}}+\frac{1}{2 L_{\mathrm{H}_{2} \mathrm{O}}} \nabla \Gamma_{\mathrm{H}_{2} \mathrm{O}}\right.\right. \\
& \left.\left.\quad+\frac{1}{2 L_{\mathrm{H}_{2}}} \nabla \Gamma_{\mathrm{H}_{2}}\right)\right]=0
\end{aligned}
$$

$$
\begin{gathered}
\rho \frac{D}{D t}\left(\Gamma_{\mathrm{O}_{2}}+\frac{1}{2} \Gamma_{\mathrm{CO}}+\frac{1}{2} \Gamma_{\mathrm{H}_{2} \mathrm{O}}+\Gamma_{\mathrm{CO}_{2}}\right) \\
-\nabla \cdot\left[\rho D _ { T } \left(\frac{1}{L_{\mathrm{O}_{2}}} \nabla \Gamma_{\mathrm{O}_{2}}+\frac{1}{2 L_{\mathrm{CO}}} \nabla \Gamma_{\mathrm{CO}}\right.\right. \\
\left.\left.+\frac{1}{2 L_{\mathrm{H}_{2} \mathrm{O}}} \nabla \Gamma_{\mathrm{H}_{2} \mathrm{O}}+\frac{1}{L_{\mathrm{CO}_{2}}} \nabla \Gamma_{\mathrm{CO}_{2}}\right)\right]=0
\end{gathered}
$$

the last three of which express element conservation for $\mathrm{C}, \mathrm{H}$, and $\mathrm{O}$, respectively, while the energy equation can be written for the thermal enthalpy $h_{T}=$ $\int^{T} c_{p} \mathrm{~d} T$ as

$$
\begin{aligned}
& \rho \frac{D}{D t}\left(h_{T}+h_{\mathrm{CH}_{4}}^{o} \Gamma_{\mathrm{CH}_{4}}+h_{\mathrm{H}_{2} \mathrm{O}}^{o} \Gamma_{\mathrm{H}_{2} \mathrm{O}}\right. \\
& \left.+h_{\mathrm{CO}}^{o} \Gamma_{\mathrm{CO}}+h_{\mathrm{CO}_{2}}^{o} \Gamma_{\mathrm{CO}_{2}}\right)-\nabla \cdot\left[\rho D _ { T } \left(\nabla h_{T}\right.\right. \\
& +\frac{h_{\mathrm{CH}_{4}}^{o}}{L_{\mathrm{CH}_{4}}} \nabla \Gamma_{\mathrm{CH}_{4}}+\frac{h_{\mathrm{H}_{2} \mathrm{O}}^{o}}{L_{\mathrm{H}_{2} \mathrm{O}}} \nabla \Gamma_{\mathrm{H}_{2} \mathrm{O}} \\
& \left.\left.+\frac{h_{\mathrm{CO}}^{o}}{L_{\mathrm{CO}}} \nabla \Gamma_{\mathrm{CO}}+\frac{h_{\mathrm{CO}_{2}}^{o}}{L_{\mathrm{CO}_{2}}} \nabla \Gamma_{\mathrm{CO}_{2}}\right)\right]=0
\end{aligned}
$$


where $h_{i}^{o}$ denotes the enthalpy of formation per mole of species $i$ and $D / D t$ is the substantial derivative. In the formulation, the Lewis number $L_{i}=\lambda /\left(\rho c_{p} D_{i}\right)$ of each species based on its diffusion coefficient $D_{i}$ will be assumed to have a constant value, different in general for different species. A low-Mach-number approximation has been employed in deriving equation 10 , where unsteady pressure variations have also been neglected, along with the effect of the differences of the specific heat at constant pressure of each species from the mean $c_{p}$. Equations 4-10 must be integrated with boundary conditions in the fuel and oxidizer streams, where conditions are identified by subscripts $f$ and $o$, respectively. For the twostream problems considered, $\Gamma_{i o}=0$ for $i \neq \mathrm{O}_{2}$ or inert (the concentration of which is determined by difference), and $\Gamma_{i f}=0$ for $i \neq \mathrm{CH}_{4}$ or inert.

\section{The Generalized Burke-Schumann Formulation}

The reaction-rate constants appearing in equation 1 are given as a function of the temperature $T$ (in kelvin) by [11] $k_{1 f}=2.2 \times 10^{4} T^{3} \exp (-4402 / T)$, $k_{2 f}=4.4 \times 10^{6} T^{1.5} \exp (373 / T), k_{2 b}=4.956 \times$ $10^{8} T^{1.5} \exp (-10796 / T)$, and $k_{3 f}=2.3 \times 10^{18} T^{-0.8}$, where $k_{1 f}, k_{2 f}$, and $k_{2 b}$ are expressed in (mole s/ $\left.\mathrm{cm}^{3}\right)^{-1}$ and $k_{3 f}$ is expressed in $\left(\text { mole }^{2} \mathrm{~s} / \mathrm{cm}^{6}\right)^{-1}$. These expressions can be used to provide estimates for the characteristic times of fuel consumption, $t_{\mathrm{F}}$ $=\left(k_{1 f}[M]\right)^{-1}$, CO oxidation, $t_{\mathrm{O}}=\left(k_{2 f}[M]\right)^{-1}$, and radical recombination, $t_{\mathrm{R}}=\left(k_{3 f}[M]^{2}\right)^{-1}$. The flame structure encountered in a particular application depends on the competition of these chemical processes with the flow field, which is characterized by a strain time $t_{\mathrm{S}}$. Evaluation of the chemical times at the adiabatic flame temperature of methane-air combustion reveals that fuel consumption is typically much faster than both $\mathrm{CO}$ oxidation and radical recombination. Because most energy release is associated with steps II and III, one can anticipate that for a flame to exist, the characteristic flow time must be of the order of or larger than $t_{\mathrm{O}}$ and $t_{\mathrm{R}}$, because if it were smaller, heat release would not occur at a sufficient rate and the temperature would readily drop leading to extinction. The relative scaling $t_{\mathrm{F}} \ll t_{\mathrm{O}} \sim t_{\mathrm{R}} \lessgtr t_{\mathrm{S}}$ indicates that fuel consumption takes place in a diffusion-controlled fashion in thin layers of characteristic thickness much smaller than the characteristic flow-field scale. These fuel-consumption sheets preclude the interdiffusion of the reactants and divide the flow field into fuel regions where $\Gamma_{\mathrm{O}_{2}}=0$ and oxidizer regions where $\Gamma_{\mathrm{CH}_{4}}=$ 0 , thereby replacing equation 4 by the algebraic condition

$$
\Gamma_{\mathrm{CH}_{4}} \Gamma_{\mathrm{O}_{2}}=0
$$

The singular behavior associated with fuel consumption was taken into account when writing the additional conservation equations 5-10. In particular, equations 6-10 were obtained from linear combinations of the original conservation equations for species and energy that do not involve the fuel-consumption rate $\omega_{\mathrm{I}}$. The form of the resulting equations suggests the use of the coupling-function variables appearing in equations $6-10$ as a replacement for the original variables $\Gamma_{i}$ s and $h_{\mathrm{T}}$. For each equation, different coupling functions appear in the Lagrangian operator and diffusion operator if the Lewis numbers of the species involved are different from unity. The coupling functions associated with the Lagrangian derivatives can be normalized to give

$$
\begin{aligned}
X= & \Gamma_{\mathrm{CH}_{4}}-\Gamma_{\mathrm{O}_{2}} \\
\mathrm{Z}_{\mathrm{C}}= & \left(\Gamma_{\mathrm{CH}_{4}}+\Gamma_{\mathrm{CO}}+\Gamma_{\mathrm{CO}_{2}}\right) / \Gamma_{\mathrm{CH}_{4} f} \\
\mathrm{Z}_{\mathrm{H}}= & \left(\Gamma_{\mathrm{CH}_{4}}+\frac{1}{2} \Gamma_{\mathrm{H}_{2} \mathrm{O}}+\frac{1}{2} \Gamma_{\mathrm{H}_{2}}\right) / \Gamma_{\mathrm{CH}_{4} f} \\
\mathrm{Z}_{\mathrm{O}}= & -\left(\Gamma_{\mathrm{O}_{2}}-\Gamma_{\mathrm{O}_{2} o}+\frac{1}{2} \Gamma_{\mathrm{CO}}\right. \\
& \left.+\frac{1}{2} \Gamma_{\mathrm{H}_{2} \mathrm{O}}+\Gamma_{\mathrm{CO}_{2}}\right) / \Gamma_{\mathrm{O}_{2} o} \\
H= & \left(h_{T}-h_{T o}+h_{\mathrm{CH}_{4}}^{o} \Gamma_{\mathrm{CH}_{4}}+h_{\mathrm{H}_{2} \mathrm{O}}^{o} \Gamma_{\mathrm{H}_{2} \mathrm{O}}\right. \\
& \left.+h_{\mathrm{CO}}^{o} \Gamma_{\mathrm{CO}}+h_{\mathrm{CO}_{2}}^{o} \Gamma_{\mathrm{CO}_{2}}\right) /\left(h_{T f}-h_{T o}\right. \\
& \left.+h_{\mathrm{CH}}^{o} \Gamma_{\mathrm{CH}_{4} f}\right)
\end{aligned}
$$

all of which are continuous but exhibit jumps in gradients across the fuel consumption sheet. The variable $X$ vanishes at this sheet and takes on the values $\Gamma_{\mathrm{CH}_{4} f}$ and $-\Gamma_{\mathrm{O}_{2} \mathrm{O}}$ at the fuel and oxidizer boundaries, respectively, exhibiting properties of the variable originally introduced by Burke and Schumann [1]. The other four variables have been normalized to vary from zero in the oxidizer stream to unity in the fuel stream. The variables $Z_{\mathrm{C}}, Z_{\mathrm{H}}$, and $Z_{\mathrm{O}}$ represent mixture-fraction variables based on the elements $\mathrm{C}, \mathrm{H}$, and $\mathrm{O}$, respectively, while the variable $H$ is an appropriately normalized enthalpy for the gas mixture.

As in previous investigations [2,3], associated with the diffusion terms in equations $6-10$, it is necessary to introduce coupling functions weighted by Lewis numbers, assumed in the present approximation to be constant. Conserved scalars for the fuel-consumption step are thus analogously defined as

$$
\begin{aligned}
& \tilde{X}=\frac{\Gamma_{\mathrm{CH}_{4}}}{L_{\mathrm{CH}_{4}}}-\frac{\Gamma_{\mathrm{O}_{2}}}{L_{\mathrm{O}_{2}}} \\
& \tilde{Z}_{\mathrm{C}}=\left(\frac{\Gamma_{\mathrm{CH}_{4}}}{L_{\mathrm{CH}_{4}}}+\frac{\Gamma_{\mathrm{CO}}}{L_{\mathrm{CO}}}+\frac{\Gamma_{\mathrm{CO}_{2}}}{L_{\mathrm{CO}_{2}}}\right) /\left(\frac{\Gamma_{\mathrm{CH}_{4} f}}{L_{\mathrm{CH}_{4}}}\right)
\end{aligned}
$$




$$
\begin{aligned}
\tilde{Z}_{\mathrm{H}}= & \left(\frac{\Gamma_{\mathrm{CH}_{4}}}{L_{\mathrm{CH}_{4}}}+\frac{1}{2} \frac{\Gamma_{\mathrm{H}_{2} \mathrm{O}}}{L_{\mathrm{H}_{2} \mathrm{O}}}+\frac{1}{2} \frac{\Gamma_{\mathrm{H}_{2}}}{L_{\mathrm{H}_{2}}}\right) /\left(\frac{\Gamma_{\mathrm{CH}_{4} f}}{L_{\mathrm{CH}_{4}}}\right) \\
\tilde{\mathrm{Z}}_{\mathrm{O}}= & -\left(\frac{\Gamma_{\mathrm{O}_{2}}}{L_{\mathrm{O}_{2}}}-\frac{\Gamma_{\mathrm{O}_{2} \mathrm{O}}}{L_{\mathrm{O}_{2}}}+\frac{1}{2} \frac{\Gamma_{\mathrm{CO}}}{L_{\mathrm{CO}}}\right. \\
& \left.+\frac{1}{2} \frac{\Gamma_{\mathrm{H}_{2} \mathrm{O}}}{L_{\mathrm{H}_{2} \mathrm{O}}}+\frac{\Gamma_{\mathrm{CO}_{2}}}{L_{\mathrm{CO}_{2}}}\right) /\left(\frac{\Gamma_{\mathrm{O}_{2} \mathrm{o}}}{L_{\mathrm{O}_{2}}}\right) \\
\tilde{H}= & \left(h_{T}-h_{\mathrm{To}_{0}}+h_{\mathrm{CH}_{4}}^{o} \frac{\Gamma_{\mathrm{CH}_{4}}}{L_{\mathrm{CH}_{4}}}\right. \\
& +h_{\mathrm{H}_{2} \mathrm{O}}^{o} \frac{\Gamma_{\mathrm{H}_{2} \mathrm{O}}}{L_{\mathrm{H}_{2} \mathrm{O}}}+h_{\mathrm{CO}}^{o} \frac{\Gamma_{\mathrm{CO}}}{L_{\mathrm{CO}}} \\
& \left.+h_{\mathrm{CO}_{2}}^{o} \frac{\Gamma_{\mathrm{CO}_{2}}}{L_{\mathrm{CO}_{2}}}\right) /\left(h_{T f}-h_{T o}+h_{\mathrm{CH}_{4}}^{o} \frac{\Gamma_{\mathrm{CH}_{4} f}}{L_{\mathrm{CH}_{4}}}\right)
\end{aligned}
$$

All of these variables and their first derivatives are continuous across the fuel-consumption layer. The variable $\tilde{X}$ achieves the values $-\Gamma_{\mathrm{O}_{2} \mathrm{o}} / L_{\mathrm{O}_{2}}$ and $\Gamma_{\mathrm{CH}_{4} f} / L_{\mathrm{CH}_{4}}$ at the oxidizer and fuel boundaries, respectively, while the other three variables are zero and unity there.

Use of the equilibrium equation 11 enables all of the variables $\Gamma_{i}$ and the thermal enthalpy to be explicitly determined in terms of $\Gamma_{\mathrm{CO}_{2}}$ and the variables defined in equation 13 through piecewise linear relationships. Equation 11 implies that the relationships for reactants are simply

$$
\begin{gathered}
\Gamma_{\mathrm{O}_{2}}=-L_{\mathrm{O}_{2}} \tilde{X}, \quad \tilde{X}<0 ; \quad \Gamma_{\mathrm{O}_{2}}=0, \quad \tilde{X}>0 \\
\Gamma_{\mathrm{CH}_{4}}=0, \quad \tilde{X}<0 ; \quad \Gamma_{\mathrm{CH}_{4}}=L_{\mathrm{CH}_{4}} \tilde{X}, \quad \tilde{X}>0
\end{gathered}
$$

while the corresponding expressions for intermediates, products, and thermal enthalpy involve a larger number of terms, an example being

$$
\begin{aligned}
\Gamma_{\mathrm{CO}}=L_{\mathrm{CO}}\left(\Gamma_{\mathrm{CH}_{4} f} / L_{\mathrm{CH}_{4}} \tilde{Z}_{\mathrm{C}}-\Gamma_{\mathrm{CO}_{2}} / L_{\mathrm{CO}_{2}}\right), \tilde{X}<0 \\
\Gamma_{\mathrm{CO}}=L_{\mathrm{CO}}\left(\Gamma_{\mathrm{CH}_{4} f} / L_{\mathrm{CH}_{4}} \tilde{Z}_{\mathrm{C}}-\tilde{X}\right. \\
\left.-\Gamma_{\mathrm{CO}_{2}} / L_{\mathrm{CO}_{2}}\right), \tilde{X}>0
\end{aligned}
$$

Because all $\Gamma_{i}$ 's as well as $h_{T}$ are determined by $\Gamma_{\mathrm{CO}_{2}}$ and the variables in equation 13 , it is evident that the five variables defined in equation 12 also are determined by these variables. In other words, the coupling functions that exhibit jumps in gradients across the fuel-consumption layer are related uniquely to those that do not through piecewise linear algebraic expressions. This is the important property that enables the generalized formulation to be implemented.

In this formulation, the species and temperature fields are determined from the $\mathrm{CO}_{2}$ conservation equation,

$$
\rho \frac{D}{D t}\left(\Gamma_{\mathrm{CO}_{2}}\right)-\nabla \cdot\left[\frac{\rho D_{T}}{L_{\mathrm{CO}_{2}}} \nabla \Gamma_{\mathrm{CO}_{2}}\right]=\omega_{\mathrm{II}}
$$

which does not involve the fast fuel-consumption step, and the generalized-mixture-fraction system

$\rho \frac{D X}{D t}-\nabla \cdot\left(\rho D_{T} \nabla \tilde{X}\right)=\omega_{\text {III }}$

$\rho \frac{D Z_{\mathrm{C}}}{D t}-\nabla \cdot\left(\rho D_{T} L_{\mathrm{CH}_{4}}^{-1} \nabla \tilde{Z}_{\mathrm{C}}\right)=0$

$\rho \frac{D Z_{\mathrm{H}}}{D t}-\nabla \cdot\left(\rho D_{T} L_{\mathrm{CH}_{4}}^{-1} \nabla \tilde{Z}_{\mathrm{H}}\right)=0$

$\rho \frac{D Z_{\mathrm{O}}}{D t}-\nabla \cdot\left(\rho D_{T} L_{\mathrm{O}_{2}}^{-1} \nabla \tilde{Z}_{\mathrm{O}}\right)=0$

$\rho \frac{D H}{D t}-\nabla \cdot\left(\rho D_{T} L_{m}^{-1} \nabla \tilde{H}\right)=0$

which results from substitution of equations 12 and 13 into equations $6-10$. In the last equation, a modified fuel Lewis number has been defined as

$$
\begin{aligned}
& L_{m}=\left(h_{T f}-h_{T o}\right. \\
& \left.+h_{\mathrm{CH}_{4}}^{0} \Gamma_{\mathrm{CH}_{4} f}\right) /\left(h_{T f}-h_{T o}+h_{\mathrm{CH}_{4}}^{o} \frac{\Gamma_{\mathrm{CH}_{4} f}}{L_{\mathrm{CH}_{4}}}\right)
\end{aligned}
$$

an expression that reduces to $L_{m}=L_{\mathrm{CH}_{4}}$ when the thermal enthalpies of the two feed streams are equal. Expressions for the mole rates of steps II and III can be obtained by substituting equations 2 and 3 into equation 1 to give

$$
\begin{gathered}
\omega_{\mathrm{II}}=k_{2 f} K_{\mathrm{OH}} \rho^{2}\left(1-k_{3 f}[M] / k_{4 f}\right)^{1 / 2} \Gamma_{\mathrm{O}_{2}}^{1 / 2} \Gamma_{\mathrm{H}_{2}}^{1 / 2} \\
\times\left[\Gamma_{\mathrm{CO}}-\left(K_{6} / K_{2}\right) \Gamma_{\mathrm{CO}_{2}} \Gamma_{\mathrm{H}_{2}} / \Gamma_{\mathrm{H}_{2} \mathrm{O}}\right] \\
\omega_{\mathrm{III}}=k_{3 f} K_{\mathrm{H}}(1- \\
\left.k_{3 f}[M] / k_{4 f}\right)^{1 / 2} \rho^{3}[M] \Gamma_{\mathrm{O}_{2}}^{3 / 2} \Gamma_{\mathrm{H}_{2}}^{3 / 2} / \Gamma_{\mathrm{H}_{2} \mathrm{O}}
\end{gathered}
$$

which are in turn functions of the variables in equation 13 through the terms $\Gamma_{i}$ 's and, more weakly, through the three-body efficiencies present in $[M]$. Note that $\omega_{\mathrm{II}}=\omega_{\mathrm{III}}=0$ when $\Gamma_{\mathrm{O}_{2}}=0$, that is, for $\tilde{X}>0$.

In the proposed formulation, the initial conservation equations for species and enthalpy transform to equations 5 and 14-18 subject to the previously stated boundary conditions, and supplemented with the algebraic equations given in equations 11-13. An interesting simplification of the formulation follows from assuming that the diffusivities of all species present in the mixture are equal to the thermal diffusivity, that is, $L_{i}=1$. In this equidiffusivity case, equations $15-18$ reduce to a single transport equation for the traditional mixture fraction $Z$, which together with equations 5 and 14 complete the description of the species and temperature fields.

In view of the resulting conservation equations, we may anticipate that slower $\mathrm{CO}$ oxidation and radical recombination do not introduce computational 


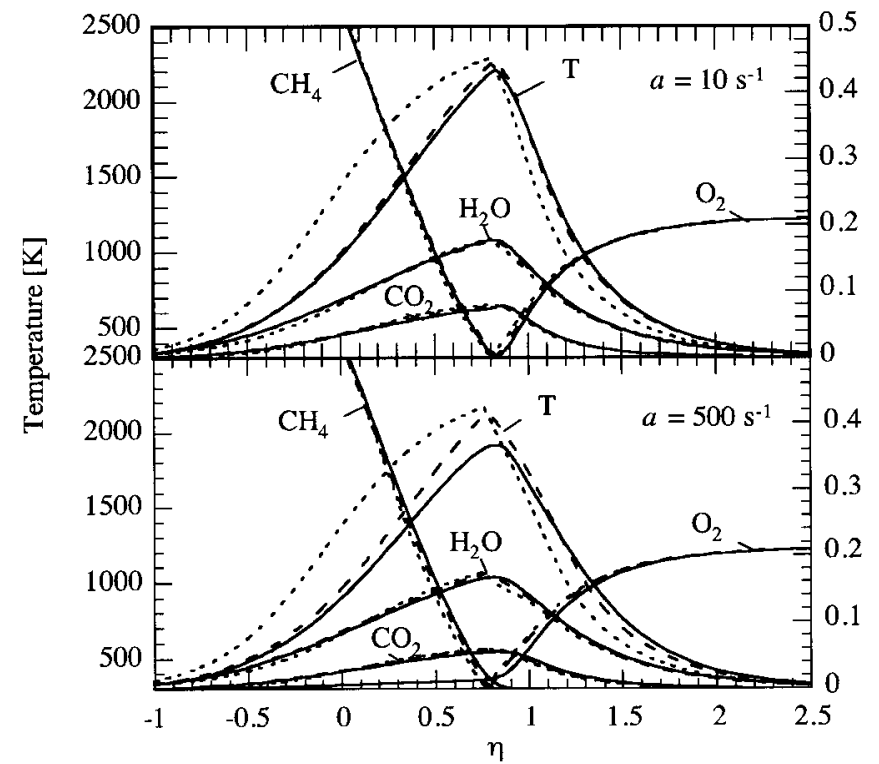

FIG. 1. The variation of the temperature and of the reactants and products mole fractions across the atmospheric counterflow mixing layer at $a=10 \mathrm{~s}^{-1}$ and at $a=500$ $\mathrm{s}^{-1}$ as obtained with detailed chemistry (solid lines), three-step reduced chemistry (dashed lines), and generalized Burke-Schumann formulation (dotted lines). difficulties because they merely decrease the magnitude of the undifferentiated source terms in equations 5 and 14. On the other hand, in the limit of fast $\mathrm{CO}$ oxidation and radical recombination, $k_{2 f} \rightarrow$ $\infty$ and $k_{3 f} \rightarrow \infty$ in equations 20 and 21, respectively, equation 21 implies that $\Gamma_{\mathrm{H}_{2}} \rightarrow 0$ in the region $\tilde{X}<$ 0 and hence everywhere (since its maximum value occurs at $\tilde{X}=0$ ), and equation 21 then implies that $\Gamma_{\mathrm{CO}} \rightarrow 0$ as well. In this limiting case, $\Gamma_{\mathrm{H}_{2}}=\Gamma_{\mathrm{CO}}$ $=0$ replaces equations 5 and 14 . The simplified form of the remaining conservation equations, which can be obtained by substituting $\Gamma_{\mathrm{H}_{2}}=\Gamma_{\mathrm{CO}}=0$ into equations 15-17, corresponds to the irreversible overall reaction $\mathrm{CH}_{4}+2 \mathrm{O}_{2} \rightarrow \mathrm{CO}_{2}+2 \mathrm{H}_{2} \mathrm{O}$. The formulation thus automatically approaches the onestep irreversible limit as in our earlier work [3].

\section{The Counterflow Diffusion Flame}

The proposed formulation is applied now to the calculation of undilute methane-air diffusion flames in the counterflow mixing layer, a test configuration for which the solution with detailed transport can be easily computed with both detailed and three-step reduced chemistry. The values $L_{\mathrm{CH}_{4}}=0.90, L_{\mathrm{O}_{2}}=$ $0.98, L_{\mathrm{CO}_{2}}=1.26, L_{\mathrm{H}_{2} \mathrm{O}}=0.73, L_{\mathrm{H}_{2}}=0.28$, and $L_{\mathrm{CO}}=1.00$ were selected for the Lewis numbers associated with the generalized Burke-Schumann formulation. For simplicity, a constant value of $c_{p}$, equal to the value at the reaction sheet of a BurkeSchumann flame, was incorporated in the definitions of $H$ and $\tilde{H}$ in equations 12 and 13 , respectively. The transport terms in equations 5 and 14-18 were written in the self-similar form corresponding to the counterflow mixing layer, which is described, for instance, in Ref. [11], where the detailed mechanism employed in the comparisons can also be found. Calculations were performed with boundary temperatures $T_{0}=T_{f}=300 \mathrm{~K}$ for values of the air-side strain rate ranging from $a=0.1 \mathrm{~s}^{-1}$ to extinction and for both atmospheric and high pressure $(p=$ 40 bar). The "FlameMaster" code [12] previously used, for instance, in Ref. [7] is utilized in the calculations, with appropriate modifications incorporated to deal with the generalized Burke-Schumann formulation.

Profiles of temperature, reactants, and products obtained with detailed and three-step reduced chemistry are compared in Fig. 1 with the results of the proposed formulation. The nondimensional selfsimilar coordinate $\eta$ used in the plot is the distance from the stagnation plane $y$ scaled according to $\eta=$ $\left[\mathrm{a} /\left(\rho_{o} \mu_{o}\right)\right]^{1 / 2} \int_{0}^{y} \rho \mathrm{d} y$, where $\mu_{o}$ is the viscosity in the airstream. As can be seen, the main inaccuracy is found in the shape of the temperature profile, with departures mainly due to the constant value of $c_{p}$ incorporated in the computations. While this value is approximately equal to that found at the fuel consumption layer, causing the peak temperature to be fairly accurate, it is clearly too small on the fuel side because of the presence of $\mathrm{CH}_{4}$, thereby leading to overpredictions for the temperature on this side, and too large on the air side because of the temperature decrease toward the boundary value, giving associated temperatures that are too low. This agreement in peak temperatures is tested for other strain conditions and also at elevated pressure in Fig. 2. Despite the increasing departures observed as extinction is approached, the simplified formulation 

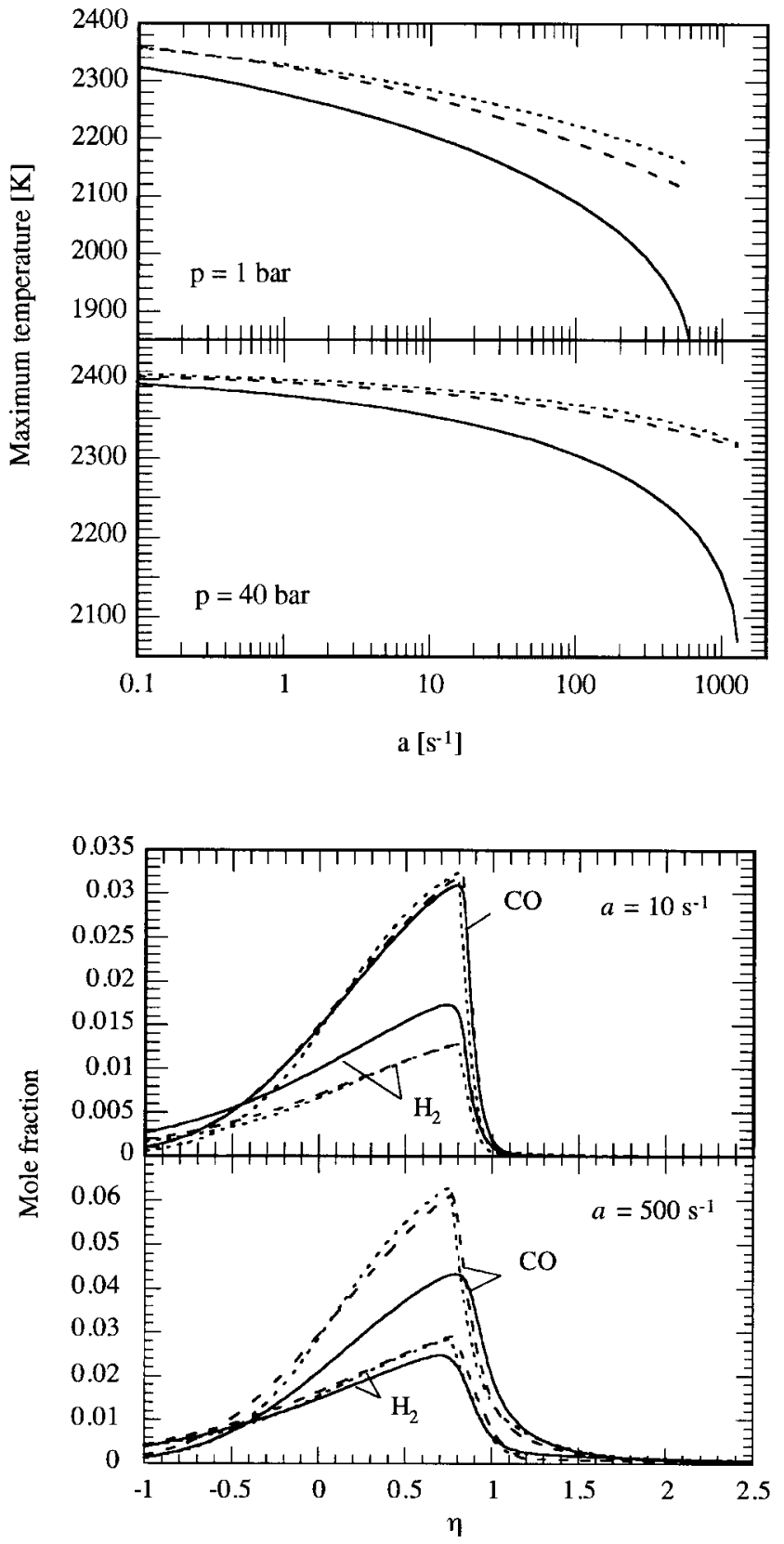

FIG. 2. The variation of the peak temperature with strain rate for $p=$ 1 bar and for $p=40$ bar as obtained with detailed chemistry (solid lines), three-step reduced chemistry (dashed lines), and generalized Burke-Schumann formulation (dotted lines).
FIG. 3. The profiles of $\mathrm{CO}$ and $\mathrm{H}_{2}$ mole fractions across the atmospheric counterflow mixing layer at $a$ $=10 \mathrm{~s}^{-1}$ and at $a=500 \mathrm{~s}^{-1}$ as obtained with detailed chemistry (solid lines), three-step reduced chemistry (dashed lines), and generalized Burke-Schumann formulation (dotted lines). provides peak temperatures with errors below $100 \mathrm{~K}$ over about 3 orders of magnitude in strain rate. It is worth pointing out that observation of fuel profiles computed with detailed chemistry reveals that no significant fuel leakage occurs near extinction, indicating that the assumption of infinitely fast fuel consumption remains valid for all strain rates.

Profiles of $\mathrm{CO}$ and $\mathrm{H}_{2}$ mole fractions associated to the flow-field conditions considered previously in
Fig. 1 are exhibited in Fig. 3, whereas Fig. 4 shows the variation of their peak values with strain rate. Figure 4 also shows peak $\mathrm{H}$-atom and $\mathrm{O}$-atom mole fractions, evaluated, respectively, using equation 2 and

$$
[\mathrm{O}]=\left(1-\frac{k_{3 f}[M]}{k_{4 f}}\right) K_{\mathrm{O}}\left[\mathrm{O}_{2}\right]\left[\mathrm{H}_{2}\right] /\left[\mathrm{H}_{2} \mathrm{O}\right]
$$




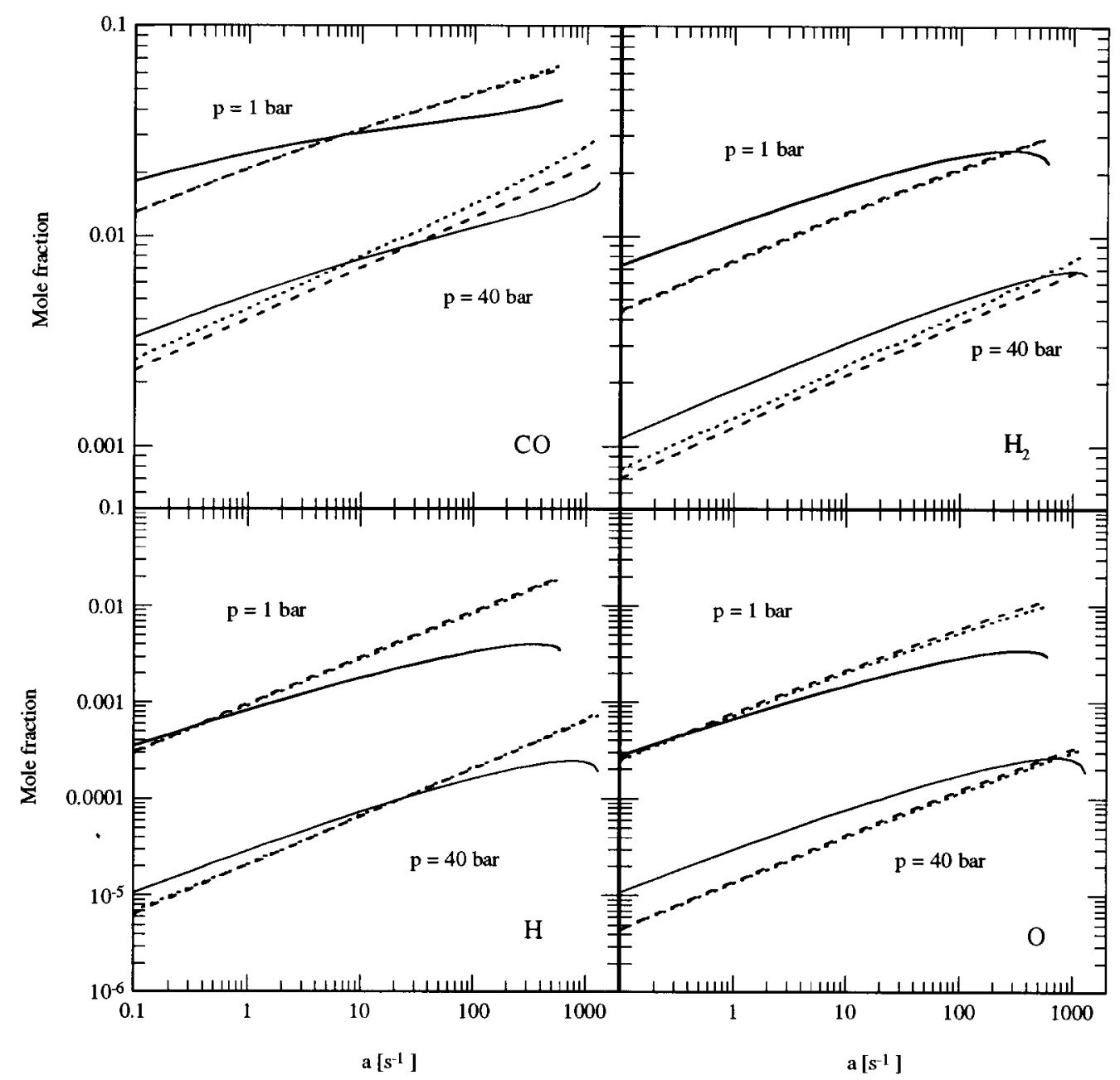

FIG. 4. Peak values of $\mathrm{CO}, \mathrm{H}_{2}, \mathrm{H}$, and $\mathrm{O}$ mole fractions as a function of the strain rate for $p=1$ bar and for $p=40$ bar as obtained with detailed chemistry (solid lines), three-step reduced chemistry (dashed lines), and generalized BurkeSchumann formulation (dotted lines).

a simplified steady-state expression involving an equilibrium constant $K_{\mathrm{O}}=K_{4} K_{6}$ that follows from the partial equilibrium assumptions for reactions 5 and 6 . As can be seen, the agreement between the results of the generalized Burke-Schumann formulation and those of the three-step reduced chemistry is very good for all intermediates, indicating that the assumption of constant $L_{i}$ introduces only minor inaccuracies in the solution. Observation of the results obtained with detailed chemistry reveals that the proposed formulation predicts peak values of $\mathrm{CO}$ and $\mathrm{H}_{2}$ with accuracies better than $30 \%$ over about 3 orders of magnitude in strain rates, with departures being somewhat larger for the $\mathrm{H}$-atom and $\mathrm{O}$ atom predictions.

\section{Discussion and Conclusions}

The continuity of the derivatives of the functions defined in equation 13 and the continuity of the functions defined in equation 12 enables finitedifference approximations to equations $14-18$ to be implemented numerically for computational purposes without front tracking. Fast fuel consumption can introduce stiffness into numerical procedures based on equations $4-10$, but equations $14-18$, together with equation 5 , remove this difficulty by retaining only the slow chemical times associated with $\omega_{\text {II }}$ and $\omega_{\text {III. }}$. The proposed formulation thus is attractive for both computational and analytical studies of diffusion flames whenever the fuel-consumption time is short compared to the flow-field time, enabling in particular the direct numerical simulation 
of flows with moderately strong nonequilibrium effects associated to flow times of the order of the times for CO oxidation and radical recombination. When the characteristic times of these two processes are comparable with those of the flow field (determined for the counterflow mixing layer computed above by the inverse of the strain rate $a^{-1}$ ), the formulation has comparable times everywhere, enhancing computational efficiency. The conservation equations 5 and 14-18 are also useful as a starting point for $k-\epsilon$ or large-eddy-simulation modeling studies.

The numerical results indicate that the formulation produces reasonable accuracy in describing methane-air diffusion flames over about 3 orders of magnitude in flow time, becoming increasingly inaccurate as extinction is approached because of the assumptions underlying the starting chemistry description. It is concluded that, while keeping the assumptions of infinitely fast fuel consumption and constant Lewis numbers, efforts to improve the generalized Burke-Schumann model must involve variable specific heats and a more complete starting chemistry model that enables in particular the description of the solution with non-negligible oxidizer leakage as the extinction regime is approached.

\section{Acknowledgments}

This collaborative work was supported by NATO under Research Grant CRG 970618. The work of M. Bollig and A. Liñán was also supported by the Spanish CICYT under Contract PB94-0400, and by INTA under Contract 40700036/1995, while that of A. L. Sánchez was also supported by the Spanish DGICYT under Contract PB95-0280, and that of F. A. Williams was also supported by the National Science Foundation through Grant CTS95-26410 and by the Office of Naval Research through Contract N0001494-1-0679.

\section{REFERENCES}

1. Burke, S. P. and Schumann, T. E. W., Ind. Eng. Chem. 20:998-1004 (1928).

2. Liñán, A. and Williams, F. A., in Fundamental Aspects of Combustion, 1st ed., Oxford University Press, New York, 1993, pp. 144-146.

3. Sánchez, A. L., Liñán, A., and Williams, F. A., Combust. Sci. Technol. 123:317-345 (1997).

4. Williams, F. A., in Modern Developments in Propulsion and Combustion (G. D. Roy, ed.), Taylor and Francis Publishers, Washington, D.C., 1997, pp. 93-128.

5. Peters, N., in Numerical Simulation of Combustion Phenomena (R. Glowinski, B. Larrouturou, and R. Temam, eds.), Lecture Notes in Physics 241, SpringerVerlag, New York, 1985, pp. 90-109.

6. Peters, N. and Kee, R. J., Combust. Flame 68:17-29 (1987).

7. Hewson, J. and Bollig, M., in Twenty-Sixth Symposium (International) on Combustion, The Combustion Institute, Pittsburgh, 1996, pp. 2171-2179.

8. Peters, N. and Williams, F. A., Combust. Flame 68:185-207 (1987).

9. Seshadri, K. and Peters, N., Combust. Flame 73:23-44 (1988).

10. Chelliah, H. K. and Williams, F. A., Combust. Flame 80:17-48 (1990).

11. Peters, N., in Reduced Kinetic Mechanisms for Applications in Combustion Systems (N. Peters and B. Rogg, eds.), Lecture Notes in Physics m15, SpringerVerlag, New York, 1993, pp. 3-14.

12. Pitsch, H. and Bollig, M., FlameMaster, A Computer Code for Homogeneous and One-Dimensional Laminar Flame Calculations, Institut für Technische Mechanik, RWTH-Aachen, 1994.

\section{COMMENTS}

George Kosály, University of Washington, USA. It appears to me that this approximation is analogous to the partial equilibrium approximation that has been used extensively to predict hydrogen-air flames. Do you expect that this approach will prove more adequate to hydrocarbon flames than it was in the hydrogen cases?

Author's Reply. Because fuel consumption is typically much faster than CO oxidation and radical recombination, we assume in the present analysis that the fuel-consumption process is infinitely fast. Following ideas first devel- oped by Burko and Schumann (Ref. [1] in the paper), the associated numerical at stiffness is then removed through the introduction of generalized coupling functions, as explained in the text. Although for simplicity in the derivation of the steady-state expressions for the radicals $\mathrm{H}, \mathrm{OH}$, and O (Equations 2, 3, and 22), the two shuffle reactions

$$
\mathrm{H}_{2}+\mathrm{O} \stackrel{5}{\rightleftharpoons} \mathrm{OH}+\mathrm{H} \text { and } \mathrm{H}_{2}+\mathrm{OH} \stackrel{6}{\rightleftharpoons} \mathrm{H}_{2} \mathrm{O}+\mathrm{H}
$$

are assumed to maintain steady state; this assumption is not intrinsic to the present approach. 